\title{
Ratio of Acceleration Time to Ejection Time of Transaortic Jet in Aortic Stenosis Depends on Acoustic Window
}

\author{
Takuya Sasaki ${ }^{1}$, Kenta Kunimutsu ${ }^{2}$, Nobuaki Tanaka ${ }^{1}$, Mayu Nakamoto ${ }^{1}$, Ayano Fujii ${ }^{2}$, \\ Tomoko Tanaka², Toru Ariyoshi², Takuya Omuro ${ }^{1}$, Ayumi Omuro ${ }^{1}$, Yasuaki Wada ${ }^{2}$, \\ Takako Maeda ${ }^{1}$, Shinichi Okuda ${ }^{1}$, and Masafumi Yano ${ }^{1}$ \\ ${ }^{1}$ Yamaguchi University School of Medicine Graduate School of Medicine \\ ${ }^{2}$ Yamaguchi University
}

January 31, 2021

\begin{abstract}
Background: Echocardiographic transaortic jet velocity (Vmax), mean pressure gradient (mPG), and aortic valve area (AVA) are routinely measured for severity of aortic stenosis (AS). Additionally, prolonged ejection time (ET), acceleration time (AT), and its ratio AT/ET are also known as indexes of AS severity. However, acoustic window dependency of AT/ET is not well studied. Methods: Eighty-one patients with AS assessed by transaortic jet tracing of all of three approaches (apical 3-chamber (3C), apical 5-chamber (5C), and right parasternal $(\mathrm{R})$ ) were included in this study. ET, AT, and AT/ET were measured on continuous Doppler recordings obtained by 3C, 5C, and R approaches. Also, ET and AT were corrected by dividing by (R-R interval) $1 / 2$, and they were named as cET and cAT. Results: No differences were observed in cET among 3 approaches. However, cAT was significantly longer in $\mathrm{R}(115+23$ msec: $\mathrm{p}<0.05)$ compared to that of $3 \mathrm{C}(105+21 \mathrm{msec})$ or $5 \mathrm{C}(105+20$ $\mathrm{msec})$. AT/ET was significantly greater in $\mathrm{R}(0.340+0.058, \mathrm{p}<0.05)$ compared to that of $3 \mathrm{C}(0.317+0.053)$ or $5 \mathrm{C}(0.316+0.055)$. AT/ET-peak V relation of $\mathrm{R}$ approach positioned significantly upward (ANCOVA, $\mathrm{p}<0.05$ ) comparing to that of $3 \mathrm{C}$ or $5 \mathrm{C}$. Also, AT/ET-AVAi relation of $\mathrm{R}$ approach positioned upward (ANCOVA, $\mathrm{p}<0.05$ ) comparing to that of $3 \mathrm{C}$ or $5 \mathrm{C}$. Conclusions: $\mathrm{AT} / \mathrm{ET}$ by $\mathrm{R}$ approach was greater than that by $3 \mathrm{C}$ or $5 \mathrm{C}$ approach. Although multiple acoustic window's approaches including $\mathrm{R}$ is recommended to obtain the maximal Vmax or $\mathrm{mPG}$, AT/ET is better in $3 \mathrm{C}$ or $5 \mathrm{C}$ approach than $\mathrm{R}$ when $\mathrm{AT} / \mathrm{ET}$ is used for AS severity.
\end{abstract}

Original Article

\section{Ratio of Acceleration Time to Ejection Time}

\section{of Transaortic Jet in Aortic Stenosis}

\section{Depends on Acoustic Window}

Takuya Sasaki MS ${ }^{1}$, Kenta Kunimitsu MS ${ }^{2}$, Nobuaki Tanaka MD ${ }^{1}$, Mayu Nakamoto $\mathrm{MS}^{1}$, Ayano Fujii RMT ${ }^{2}$, Tomoko Tanaka $\mathrm{RMT}^{2}$, Toru Ariyoshi $\mathrm{MS}^{2}$,

Takuya Omuro $\mathrm{MS}^{1}$, Ayumi Omuro MD ${ }^{3}$, Yasuaki Wada $\mathrm{MD}^{2}$,

Takako Maeda $\mathrm{MD}^{3}$, Shinichi Okuda $\mathrm{MD}^{3}$, Masafumi Yano MD ${ }^{3}$

1: Department of Clinical Laboratory Sciences, Yamaguchi University Graduate School of Medicine, Ube, Japan

2: Division of Laboratory, Yamaguchi University Hospital, Ube, Japan 
3: Department of Medicine and Clinical Sciences, Yamaguchi University Graduate School of Medicine, Ube, Japan

Correspondence: Nobuaki Tanaka MD

Mailing address:

Department of Clinical Laboratory Sciences

Yamaguchi University Graduate School of Medicine

1-1-1 Minami-Kogushi

Ube, 755-8505 Yamaguchi, Japan

Tel +81-836-22-2807

Fax $+81-836-22-2130$

E-mail:nktanaka@yamaguchi-u.ac.jp

Running head: AT/ET in AS Depends on Acoustic Window

Funding: None.

\section{ABSTRACT}

Background: Echocardiographic transaortic jet velocity (Vmax), mean pressure gradient (mPG), and aortic valve area (AVA) are routinely measured for severity of aortic stenosis (AS). Additionally, prolonged ejection time (ET), acceleration time (AT), and its ratio AT/ET are also known as indexes of AS severity. However, acoustic window dependency of AT/ET is not well studied.

Methods: Eighty-one patients with AS assessed by transaortic jet tracing of all of three approaches (apical 3-chamber (3C), apical 5-chamber (5C), and right parasternal (R)) were included in this study. ET, AT, and AT/ET were measured on continuous Doppler recordings obtained by $3 \mathrm{C}, 5 \mathrm{C}$, and $\mathrm{R}$ approaches. Also, ET and AT were corrected by dividing by (R-R interval $)^{1 / 2}$, and they were named as cET and cAT.

Results: No differences were observed in cET among 3 approaches. However, cAT was significantly longer in $\mathrm{R}(115+23 \mathrm{msec}: \mathrm{p}<0.05)$ compared to that of $3 \mathrm{C}(105+21 \mathrm{msec})$ or $5 \mathrm{C}(105+20 \mathrm{msec})$. AT/ET was significantly greater in $\mathrm{R}(0.340+0.058, \mathrm{p}<0.05)$ compared to that of $3 \mathrm{C}(0.317+0.053)$ or $5 \mathrm{C}(0.316+0.055)$. AT/ET-peak V relation of R approach positioned significantly upward (ANCOVA, $<<0.05$ ) comparing to that of $3 \mathrm{C}$ or $5 \mathrm{C}$. Also, AT/ET-AVAi relation of $\mathrm{R}$ approach positioned upward (ANCOVA, $\mathrm{p}<0.05$ ) comparing to that of $3 \mathrm{C}$ or $5 \mathrm{C}$.

Conclusions: AT/ET by $\mathrm{R}$ approach was greater than that by $3 \mathrm{C}$ or $5 \mathrm{C}$ approach. Although multiple acoustic window's approaches including $\mathrm{R}$ is recommended to obtain the maximal Vmax or $\mathrm{mPG}$, AT/ET is better in $3 \mathrm{C}$ or $5 \mathrm{C}$ approach than $\mathrm{R}$ when $\mathrm{AT} / \mathrm{ET}$ is used for AS severity.

Keywords: aortic stenosis, systolic and diastolic time intervals, Doppler ultrasound,

acoustic window

\section{Introduction}

Echocardiography is the essential tool for assessing the severity of aortic stenosis (AS) ${ }^{1-5}$ Peak transaortic valve jet velocity (Vmax), mean transaortic valve pressure gradient ( $\mathrm{mPG}$ ), and aortic valve area (AVA) are the main indexes of AS severity. ${ }^{3,4}$ However, one or two indexes of them may sometimes discord with each other, and require another solution. One of the possible situations may be seen in case of depressed left ventricular (LV) function. In case of AS with depressed LV function, Vmax, mean PG, and stenotic AVA are underestimated because of reduced stroke volume (SV) known as low-flow low-gradient (LFLG) AS. ${ }^{6}$ 
In such cases, dobutamine stress echocardiography is recommended for correct diagnosis of the severity of AS. ${ }^{3,4,7}$

Ratio of acceleration time (AT) to ejection time (ET) of transaortic flow, i.e. AT/ET, is reported as a reliable index of severity of AS. ${ }^{8-12} \mathrm{AT} / \mathrm{ET}$ is positively correlated with Vmax, mean PG, and negatively correlated with AVA or indexed AVA (AVAi). Also, AT/ET is proposed as an alternative to detect prosthetic valve malfunction caused by stenosis. ${ }^{13}$

To obtain the appropriate Vmax and $\mathrm{mPG}$ in patients with AS, approaching by multiple acoustic windows are recommended by ASE guideline. ${ }^{4}$ However, the acoustic window dependency on the measurement of AT/ET have not been well studied yet, therefore, we investigated the effect of different approach on AT/ET in patients with AS.

\section{Materials and Methods}

Eighty-one patients with AS who visited Yamaguchi University Hospital from January 2014 to July 2018 for assessment of AS severity by echocardiography with multiple approach (apical 3-chamber (3C), apical 5-chamber $(5 \mathrm{C})$, and right parasternal $(\mathrm{R})$ ) were included in this retrospective study. Echocardiographic images and data were retrieved from database and clinical chart and assessed.

These echocardiographic data were obtained by one of the following equipment: Vivid 7 (GE Healthcare), EPIC (Philips), Aplio 500 (Canon Medical Systems), or Aplio i900 (Canon Medical Systems) by standard examination technique.

Inclusion criteria were 1) patients with AS > mild, 2) LVEF $>50 \%$ and stroke volume index (SVI) $>$ $35 \mathrm{~mL} / \mathrm{m}^{2}$. Sex and age were not limited. Exclusion criteria were 1) congenital heart disease other than bicuspid aortic valve, 2) arrhythmia (ventricular tachycardia, supraventricular tachycardia, atrial fibrillation, and so on) at the time of echocardiographic examination, 3) post-operative state of valvular disease, and 4) severe valvular heart disease other than AS.

Geographic data were collected including sex, age, height, body weight, complications, past medical history, history of present illness, and treatment until the occasion of the echocardiographic examination. Echocardiographic data were LV internal dimension in end-diastole (LVDd), LV internal dimension in end-systole (LVDs), LV ejection fraction (LVEF), interventricular thickness (IVS), LV posterior thickness (PW), LV mass index (LVMI), SVI, and AVAi. Heart rate (HR), systolic blood pressure (SBP), and diastolic blood pressure (DBP) were at the occasion of echocardiographic examination.

The AT, ET, and AT/ET as well as peak transaortic Vmax were analyzed from transaortic Doppler recordings of $3 \mathrm{C}, 5 \mathrm{C}$, and $\mathrm{R}$ approaches (Figure $\mathbf{1}$ ). Both of ET and AT were corrected according to HR by dividing by (R-R interval $)^{1 / 2}$, and they were named as cET and cAT. AVA was obtained by continuous equation using an approach of which transaortic Vmax was the highest of all approaches.

All of measurements of time interval were average of 3 consecutive beats. Sweep speed on the screen for measurements were used as $50-100 \mathrm{~mm} / \mathrm{sec}$, depending on the HR of the patients. Doppler gain was adjusted properly in each patient, and it was kept constant within the patient.

Intra-observer variability and inter-observer variability of time measurements: Intra-observer variability of AT, ET, and AT/ET were assessed by regression analysis and Bland-Altman plot. ${ }^{14,15}$ Total of 90 beats (each 9 beats from 10 cases) was measured once, then the same 90 beats were measured on the other day by the same investigator for the intra-observer variability.

Inter-observer variability of AT, ET, and AT/ET were also assessed. Total of 150 beats (51 beats of 3C, 51 beats of $5 \mathrm{C}$ and 48 beats of $\mathrm{R}$ from 38 cases) were measure by the $1^{\text {st }}$ investigator, then the same beats were re-measured by the $2^{\text {nd }}$ investigator as the blinded to the $1^{\text {st }}$ measurements. Then, to compare the data of the apical and $\mathrm{R}$ approach, 51 beats of $\mathrm{R}$ data were added for analysis. Finally, 102 beats of apical approach ( 51 beats of $3 \mathrm{C}$ and 51 beats of $5 \mathrm{C}$ ) and 99 beats of $\mathrm{R}$ approach were analyzed by regression analysis and Bland-Altman plot. 
Statistical analysis: Continuous variables are expressed as mean + SD. Commercially available software (StatFlex ver.6, Artech Co. Ltd.) was used for statistical analysis. Analysis of variance (ANOVA) and post-hoc test (Dunn) was used for multiple comparison. Analysis of covariance (ANCOVA) was used for comparison of regression lines (JUMP pro Windows, ver 13). Bland-Altman plot was used for intra- and inter-observer variability. $\mathrm{P}<0.05$ was assumed as statistical significance.

This study was approved by an Institutional Review Board of Yamaguchi University Hospital (H30-041-[1]). The Institutional Review Board approved this retrospective analysis of clinically acquired data and waived the need for written informed consent.

\section{Results}

Patients' backgrounds $(\mathrm{n}=81)$ are shown in Table $\mathbf{1}$. Mean age was $79+10$ year-old. Female patients (57\%) were more than male patients (43\%). Echocardiographic data were also shown in Table 1 . LV was not dilated, LVMI was increased due to AS, and LVEF and SVI was preserved because of inclusion criteria. AVAi by continuous equation was limited $\left(0.63+0.25 \mathrm{~cm}^{2} / \mathrm{m}^{2}\right)$ and peak $\mathrm{V}$ was increased $(4.0+1.1 \mathrm{~m} / \mathrm{sec})$. Distribution of AS severity was as follows: 17 cases of mild AS, 19 cases of moderate AS, and 45 cases of severe AS.

Peak velocity of 3 approaches: Mean values of peak $\mathrm{V}$ were $3.65+1.06 \mathrm{~m} / \mathrm{sec}$ by $3 \mathrm{C}, 3.65+1.06 \mathrm{~m} / \mathrm{sec}$ by $5 \mathrm{C}$, and $3.83+1.17 \mathrm{~m} / \mathrm{sec}$ by $\mathrm{R}$, namely, peak $\mathrm{V}$ of $\mathrm{R}$ approach was slightly greater than other approaches (Figure 2, ns).

cET, cAT, and AT/ET of 3 approaches: Figure 3 shows cET, cAT, and AT/ET obtained from 3C, 5C, and $\mathrm{R}$ approaches. $\mathrm{cET}$ was no significant difference between approaches. cAT was significantly longer in $\mathrm{R}$ $(115+23 \mathrm{msec})$ than $3 \mathrm{C}(105+21 \mathrm{msec})$ and $5 \mathrm{C}$ approaches $(105+20 \mathrm{msec})(\mathrm{p}<0.05)$. Therefore, AT/ET was significantly greater in $\mathrm{R}(0.34+0.058: \mathrm{p}<0.05)$ than $3 \mathrm{C}(0.317+0.053)$ and $5 \mathrm{C}(0.316+0.055)$.

AT/ET and severity of AS 1) AT/ET-peak V relation: Next, we analyzed the relation between AT/ET and severity of AS. First, we plotted AT/ET-peak V relation obtained by 3C, 5C and R approach (Figure 4 ). For each approach, linear regression analysis was performed. Regression equations were $\mathrm{y}=0.0341 \mathrm{x}+0.1862$ for $3 \mathrm{C}, \mathrm{y}=0.0364 \mathrm{x}+0.1750$ for $5 \mathrm{C}$, and $\mathrm{y}=0.0374 \mathrm{x}+0.1887$ for $\mathrm{R}$, respectively. Regression line for $\mathrm{R}$ was positioned significantly higher than that of $3 \mathrm{C}$ or $5 \mathrm{C}$ by ANCOVA $(\mathrm{p}<0.05)$.

AT/ET and severity of AS 2) AT/ET-AVAi relation: Then, we plotted AT/ET-AVAi relation (Figure 5 ). For each approach, linear regression analysis was performed. Regression equation was $\mathrm{y}=-0.1763 \mathrm{x}+0.4314$ for $3 \mathrm{C}, \mathrm{y}=-0.1824 \mathrm{x}+0.4337$ for $5 \mathrm{C}$, and $\mathrm{y}=-0.1753 \mathrm{x}+0.4480$ for $\mathrm{R}$, respectively. Regression line for $\mathrm{R}$ was also positioned significantly higher than that of $3 \mathrm{C}$ or $5 \mathrm{C}$ by ANCOVA $(\mathrm{p}<0.05)$.

ROC analysis of 3 approaches: Therefore, we obtained 3 ROC curves of each approach to differentiate severe AS from moderate AS (Figure 6 ). According to the ROC analysis, cutoff AT/ET values for moderate and severe AS were $0.305(\mathrm{AUC}=0.94)$ by $3 \mathrm{C}$ approach, $0.308(\mathrm{AUC}=0.94)$ for $5 \mathrm{C}$ approach, and 0.331 (AUC $=0.91)$ for $\mathrm{R}$ approach, respectively.

Intra-observer variability of time measurements:Regression coefficients of AT were 0.994, 0.990, and 0.997 for 3C, 5C, and R, respectively. Regression coefficients of ET were 0.996, 0.997, and 0.990 for 3C, 5C, and R, respectively. Regression coefficients of AT/ET were $0.993,0.991$, and 0.998 for 3C, 5C, and R, respectively.

Concerning to the mean of difference $(+2 \mathrm{SD})$ of AT were $0.4+1.8 \mathrm{msec},-0.3+2.7 \mathrm{msec}$, and $0.5+1.5 \mathrm{msec}$ for 3C, $5 \mathrm{C}$, and R, respectively. Mean of difference $(+2 \mathrm{SD})$ of ET were $0.4+2.4 \mathrm{msec},-0.5+2.6 \mathrm{msec}$, and $1.0+3.5 \mathrm{msec}$ for $3 \mathrm{C}, 5 \mathrm{C}$, and $\mathrm{R}$, respectively. Mean of difference $(+2 \mathrm{SD})$ of $\mathrm{AT} / \mathrm{ET}$ were $-0.002+0.005$, $-0.001+0.007$, and $0.001+0.003$ for $3 \mathrm{C}, 5 \mathrm{C}$, and $\mathrm{R}$, respectively.

Inter-observer variability of time measurements:Although inter-observer variability was slightly larger than that of intra-observer variability, the measurement errors were considered within the clinically accepted ranges. Regression coefficients of AT were $0.910,0.880$, and 0.914 for $3 \mathrm{C}, 5 \mathrm{C}$, and R, respectively. Regression 
coefficients of ET were $0.951,0.969$, and 0.892 for $3 \mathrm{C}, 5 \mathrm{C}$, and R, respectively. Regression coefficients of $\mathrm{AT} / \mathrm{ET}$ were $0.902,0.837$, and 0.880 for $3 \mathrm{C}, 5 \mathrm{C}$, and $\mathrm{R}$, respectively.

Concerning to the mean of difference $(+2 \mathrm{SD})$ of AT were $0.8+7.8 \mathrm{msec},-2.7+9.2 \mathrm{msec}$, and $-1.6+9.9 \mathrm{msec}$ for $3 \mathrm{C}, 5 \mathrm{C}$, and $\mathrm{R}$, respectively. Mean of difference $(+2 \mathrm{SD})$ of ET were $-1.1+10.4 \mathrm{msec},-0.1+8.0 \mathrm{msec}$, and $0.9+15.7 \mathrm{msec}$ for $3 \mathrm{C}, 5 \mathrm{C}$, and R, respectively. Mean of difference $(+2 \mathrm{SD})$ of AT/ET were $0.003+$ $0.020,0.008+0.027$, and $0.007+0.027$ for $3 \mathrm{C}, 5 \mathrm{C}$, and $\mathrm{R}$, respectively.

Additionally, comparison between apical (3C and 5C) and R approach was done (Figure 7 ). By 102 beats $(3 \mathrm{C}$ and $5 \mathrm{C})$ analysis, $\mathrm{r}=0.893(\mathrm{p}<0.001), \mathrm{CV}=6.76 \%$ for $\mathrm{AT} ; \mathrm{r}=0.957(\mathrm{p}<0.001), \mathrm{CV}=2.02 \%$ for ET; $\mathrm{r}=0.867(\mathrm{p}<0.001), \mathrm{CV}=6.11 \%$ for AT $/ \mathrm{ET}$. Then, by 99 beats $(\mathrm{R})$ analysis, $\mathrm{r}=0.849(\mathrm{p}<0.001), \mathrm{CV}=8.33 \%$ for AT; $\mathrm{r}=0.887(\mathrm{p}<0.001), \mathrm{CV}=3.05 \%$ for $\mathrm{ET} ; \mathrm{r}=0.826(\mathrm{p}<0.001), \mathrm{CV}=7.92 \%$ for AT $/ \mathrm{ET}$.

Inter-observer variability of peak $\mathrm{V}(\mathrm{Vmax})$ : We also checked the inter-observer variability in both of apical approach (3C and $5 \mathrm{C}$ ) and $\mathrm{R}$ approach. Concerning to the regression coefficients of peak $\mathrm{V}$ in apical approach was $0.997(\mathrm{n}=32, \mathrm{p}<0.01)$, and that in $\mathrm{R}$ approach was $0.980(\mathrm{n}=29, \mathrm{p}<0.01)$. The $\mathrm{CV}$ was $3.51 \%$ for apical approach, and $3.41 \%$ for $\mathrm{R}$ approach, respectively, thus inter-observer variability of Vmax was no significant difference between apical and right parasternal approach. They were also considered to be within the clinically accepted ranges.

\section{Discussion}

According to the recent progress in treatment of $\mathrm{AS}^{16}$, more accurate and adequate assessment is required for the severity of AS from the prognostic aspect. ${ }^{17,18}$ Echocardiography is the main tool for assessing AS to obtain Vmax, mPG, and AVA as the major indexes of the severity of AS non-invasively and repeatedly. ${ }^{3,4}$ However, one or two indexes of them may sometimes discord with each other, and then require alternative solution. One of the possible situations was in case of LFLG AS, which requires dobutamine stress echocardiography for correct diagnosis ${ }^{3-7}$, though we did not include such cases in this study. Another situation may be due to technical difficulty, not able to obtain good echocardiographic images, suboptimal Doppler signals with ambiguous envelope in the real world.

Here, the ratio of AT to ET of transaortic Doppler flow signal, i.e. AT/ET, is recently reported as a reliable index of AS severity ${ }^{8-12}$, and positively correlated with peak $\mathrm{V}, \mathrm{mPG}$, and negatively correlated with AVA or AVAi. This index has potential to be added as a useful information of AS severity in such cases.

Some investigators have already reported that the usefulness of AT/ET. Nakamura et al reported that AT/ET correlated well with peak V and catheter derived $\mathrm{mPG}$ in $1987 .{ }^{8}$ Zekry reported AT/ET was useful in assessing dysfunction of prosthetic valve in aortic valve position. ${ }^{13}$ AT/ET is an index of easy measurement, easy recognition by eyes, and more importantly it is independent from the transaortic flow rate. Therefore, AT/ET is useful in differentiating true severe LFLG AS and pseudo AS, as Kamimura et al ${ }^{11}$ and Gamaza-Chulián ${ }^{12}$ reported. Griguer et al reported that prognosis was poor in patients with severe AS of $\mathrm{AT} / \mathrm{ET}>0.36 .^{19}$

However, these reports were based on the data acquired from no matter which acoustic window of the fastest transaortic velocity for a single patient with AS or they assumed as the identity of the AT/ET obtained from any acoustic windows. To obtain the true peak $\mathrm{V}$ of patients with AS, multiple acoustic windows approaching is recommended, while time interval measurement for $\mathrm{AT} / \mathrm{ET}$ is able in apical or right parasternal acoustic window. ${ }^{20,21}$ However, the results of time interval measurement obtained from each acoustic window have not been compared so far, therefore, we investigated the effects of acoustic windows or approaching on AT/ET measurement by measuring AT, ET, and AT/ET on continuous Doppler transaortic flow waves acquired from apical (3C and $5 \mathrm{C}$ ) and $\mathrm{R}$ acoustic windows in the same patients, and found the non-uniform results between different acoustic windows.

In this study, there was no significant difference in cET between apical (3C and $5 \mathrm{C}$ ) and $\mathrm{R}$ approach in patients with AS. However, cAT was significantly greater in R approach than apical approach, then AT/ET was also greater in $\mathrm{R}$ approach than apical approach. 
There are some possible reasons for greater AT/ET by R approach than apical approach. One is the difference in peak V caused by approaches. Thaden et al reported that the highest peak V of patients with severe AS was achieved by R approach in nearly half of their cases. ${ }^{22}$ Since severe AS was also more than half in our 81 patients in our study and the higher peak $\mathrm{V}$ was obtained by $\mathrm{R}$ approach rather than apical approach (3C or $5 \mathrm{C}$ ), time interval difference from earlier suboptimal peak $\mathrm{V}$ to later true peak $\mathrm{V}$ might cause greater AT measurement value.

Another possibility was an error in time-interval measurement caused by $\mathrm{R}$ approach. A flow characteristic of $\mathrm{R}$ approach is turbulent signal in Valsalva sinus after severely restricted aortic valve as recognized by color Doppler image. We cannot exclude the possibility of the effect of the turbulent flow signal on the envelope of the continuous wave Doppler signal which may make difficult to recognize the exact timing of the peak velocity without clear envelope of Doppler signal. Because of this reason, the variability of the inter-observer measurement difference was greater in $\mathrm{R}$ approach than in apical approach. Therefore, we need to pay an attention to measure AT/ET by R approach, though it is superior in peak V detection by $\mathrm{R}$ approach to apical approach.

Additionally, we consider the effect of HR on ET or AT measurement since ET or AT is affected by HR. Zoghbi et al ${ }^{23}$ reported that ET shortened by $15 \mathrm{msec}$ as $10 \mathrm{bpm}$ increase in HR in patients with AS in HR range of 55-110 bpm. Also, Reant et $\mathrm{al}^{24}$ reported that ET shortened by $15 \mathrm{msec}$ per $10 \mathrm{bpm}$ increase in HR in AS with reduced LV systolic function. However, the ratio AT/ET itself is reported to be independent of $\mathrm{HR}^{19}$, thus the effect of HR on AT/ET may be minor.

Finally, AT/ET is supposed to be a useful index of AS severity because this is easy to measure, easy to recognize, and applicable to LFLG AS. ${ }^{12}$ However, it needed to pay attention to the effect of acoustic windows on the decision of AS severity by AT/ET as revealed in the study.

Limitation of study: This study did not include AS patients with decreased LVEF so-called LFLG AS, or patients with atrial fibrillation, or patient with other significant valvular lesions. The exact cause of the AT/ET discrepancy has not been determined in the clinical situation though possible cause has mentioned in the text.

\section{Conclusion}

$\mathrm{AT} / \mathrm{ET}$ is a reliable and useful index for AS severity. However, AT/ET values obtained by the right parasternal approach is slightly greater than those obtained by apical approach, which should be called an attention to prevent incorrect diagnosis of AS severity. The right parasternal approach is useful to detect the highest peak transaortic velocity, however, AT/ET values obtained from the right parasternal approach were not always as same as those obtained from the apical approach.

Acknowledgement: We thank Ms Nakaya S, Mr Uechi T, and Ms Katamoto Y for their help in analysis of data variability.

\section{References}

1. Chambers JB. Aortic stenosis. Eur J Echocardiogr 2009;10:i11-9.

2. Lindroos M, Kupari M, Heikkilä J, et al. Prevalence of aortic valve abnormalities in the elderly: an echocardiographic study of a random population sample. J Am Coll Cardiol 1993;21:1220-5.

3. Saikrishnan N, Kumar G, Sawaya FJ, et.al. Accurate assessment of aortic stenosis: a review of diagnostic modalities and hemodynamics. Circulation 2014;129:244-53.

4. Baumgartner H, Hung J, Bermejo J, et al. Recommendations on the echocardiographic assessment of aortic valve stenosis: a focused update from the European association of cardiovascular imaging and the American society of echocardiography. J Am Soc Echocardiogr 2017;30:372-92.

5. Nishimura RA, Otto CM, Bonow RO, et al. 2017 AHA/ACC focused update of the 2014 AHA/ACC guideline for the management of patients with valvular heart disease: a report of the American College 
of Cardiology/American Heart Association Task Force on clinical practice guidelines. J Am Coll Cardiol 2017;70:252-89.

6. Awtry E, Davidoff R. Low-flow/low-gradient aortic stenosis. Circulation 2011;124:e739-41.

7. Annabi MS, Touboul E, Dahou A, et al. Dobutamine stress echocardiography for management of low-flow low-gradient aortic stenosis. J Am Coll Cardiol 2018;71:475-85.

8. Nakamura K, Shiina T, Satomi G, et al. Severity of aortic stenosis assessed by Doppler techniques. J Cardiol 1987;17:807-16.

9. Kim SH, Kim JS, Kim BS, et al. Time to peak velocity of aortic flow is useful in predicting severe aortic stenosis. Int J Cardiol 2014; 172: e443-6.

10. Gamaza-Chulián S, Camacho-Freire S, Toro-Cebada R, et al. Ratio of acceleration time to ejection time for assessing aortic stenosis severity. Echocardiography 2015; 32: 1754-61.

11. Kamimura D, Hans S, Suzuki T, et al. Delayed time to peak velocity is useful for detecting severe aortic stenosis. J Am Heart Assoc 2016; 5: e003907. doi: 10.1161/JAHA.116.003907

12. Gamaza-Chulián S, Díaz-Retamino E, Camacho-Freire S, et al. Acceleration time and ratio of acceleration time to ejection time in aortic stenosis: new echocardiographic diagnostic parameter. J Am Soc Echocardiogr 2017;30:947-55.

13. Zekry SB, Saad RM, Özkan M, et al. Flow acceleration time and ratio of acceleration time to ejection time for prosthetic aortic valve function. J Am Coll Cardiol Img 2011;4:1161-70.

14. Bland JM, Altman DG. Statistical methods for assessing agreement between two methods of clinical measurement. Lancet 1986; 327: 307-10.

15. Giavarina D. Understanding Bland Altman analysis. Biochemia Medica 2015;25:141-51.

16. Mack MJ, Leon MB, Smith CR, et al. 5-year outcomes of transcatheter aortic valve replacement or surgical aortic valve replacement for high surgical risk patients with aortic stenosis (PARTNER 1): a randomized controlled trial. Lancet 2015; 385: 2477-2484.

17. Rosenhek R, Zilberszac R, Schemper M, et al. Natural history of very severe aortic stenosis. Circulation 2010;121:151-156.

18. Varadarajan P, Kapoor N, Bansal RC, et al. Survival in elderly patients with severe aortic stenosis is dramatically improved by aortic valve replacement: results from a cohort of 277 patients aged $>80$ years. Eur J Cardiothorac Surg 2006;30:722-7.

19. Griguer AR, Tribouilloy C, Truffer A, et al. Clinical significance of ejection dynamics parameters in patients with aortic stenosis: an outcome study. J Am Soc Echocardiogr 2018;31:51-60.

20. Hatle L, Angelsen BA, Tromsdal A. Non-invasive assessment of aortic stenosis by Doppler ultrasound. Br Heart J 1980;43:284-92.

21. Williams GA, Labovitz AJ, Nelson JG, et al. Value of multiple echocardiographic views in the evaluation of aortic stenosis in adults by continuous-wave Doppler. Am J Cardiol 1985;55: 445-9.

22. Thaden JJ, Nkomo VT, Lee KJ, et al. Doppler imaging in aortic stenosis: the importance of the nonapical imaging windows to determine severity in a contemporary cohort. J Am Soc Echocardiogr 2015;28:780-5.

23. Zoghbi WA, Galan A, Quinones MA. Accurate assessment of aortic stenosis severity by Doppler echocardiography independent of aortic jet velocity. Am Heart J 1988; 855-63.

24. Reant P, Dijos M, Donal E, et al. Systolic time intervals as simple echocardiographic parameters of left ventricular systolic performance: correlation with ejection fraction and longitudinal two-dimensional strain. Eur J Echocardiogr 2010; 11: 834-44. 


\section{Table 1. Patients' characteristics}

Background $(\mathrm{n}=81)$

\begin{tabular}{ll}
\hline Age, year-old & $79+10$ \\
Sex $($ male/female $)$ & $35(43 \%) / 46(57 \%)$ \\
Heart rate, bpm & $65+10$ \\
SBP, mmHg & $138+20$ \\
DBP, mmHg & $72+11$ \\
BSA, m ${ }^{2}$ & $1.51+0.18$ \\
Hypertension & $65(80 \%)$ \\
Dyslipidemia & $46(57 \%)$ \\
Diabetes mellitus & $17(21 \%)$ \\
Echocardiographic data & Echocardiographic data \\
LVDd, mm & $47+5$ \\
LVDs, mm & $29+6$ \\
LVEF, $\%$ & $67+8$ \\
IVS, mm & $11+2$ \\
PW, mm & $10+1$ \\
LVMI, g/m $/ \mathrm{m}^{2}$ & $114+27$ \\
SVI, mL $/ \mathrm{m}^{2}$ & $51+9$ \\
Peak $\mathrm{V}, \mathrm{m} / \mathrm{s}$ & $4.0+1.1$ \\
AVAi, $\mathrm{cm}^{2} / \mathrm{m}^{2}$ & $0.63+0.25$ \\
\hline
\end{tabular}

SBP, systolic blood pressure; DBP, diastolic blood pressure; BSA, body surface area;

LVDd, left ventricular end-diastolic diameter; LVDs, left ventricular end-systolic diameter; LVEF, left ventricular ejection fraction; IVS, interventricular septum thickness; PW, posterior wall thickness; LVMI, left ventricular mass index; SVI, stroke volume index; peak V, peak velocity of transaortic valve; AVAi, aortic valve area index.

Figure legends

Figure1 Measurement of AT and ET on the transaortic valve Doppler tracing from different acoustic windows. $3 \mathrm{C}$ : apical 3 chamber view, $5 \mathrm{C}$ : apical 5 chamber view, R: right parasternal approach. AT is the period between the onset of transaortic flow and the peak of it. ET is the period between the onset of transaortic flow and the termination of it. For both AT and ET, 3 consecutive beats were measured and averaged. cAT and cET were obtained by dividing AT and ET by square root of R-R interval.

Figure 2 Comparison of peak transaortic velocity of different approach.

3C: apical 3 chamber view, 5C: apical 5 chamber view, R: right parasternal approach.

Figure 3 cET, cAT, and AT/ET by different approach. cET was no significant different between 3 different approaches. cAT was prolonged in $\mathrm{R}$ approach compared to $3 \mathrm{C}$ or $5 \mathrm{C}$ approach. AT/ET was greater in $\mathrm{R}$ approach than that of $3 \mathrm{C}$ or $5 \mathrm{C}$ approach. 3C: apical 3 chamber view, $5 \mathrm{C}$ : apical 5 chamber view, R: right parasternal approach.

Figure 4 AT/ET-peak $\mathrm{V}$ relation in $3 \mathrm{C}, \mathbf{5 C}$, and $\mathrm{R}$ approaches.AT/ET-peak V relation of $\mathrm{R}$ approach was significantly upward shifted form $3 \mathrm{C}$ or $5 \mathrm{C}$ approach $(\mathrm{p}<0.05)$.

Figure 5 AT/ET-AVAi relation in 3C, 5C, and $\mathrm{R}$ approaches.AT/ET-AVAi relation of $\mathrm{R}$ approach was significantly upward shifted form $3 \mathrm{C}$ or $5 \mathrm{C}$ approach $(\mathrm{p}<0.05)$.

Figure 6 ROC analysis of AT/ET cutoff value for severe AS. Left (A): by using AT/ET of 3C, cutoff value was $0.305(\mathrm{AUC}=0.094)$, middle $(\mathrm{B})$ : by using $\mathrm{AT} / \mathrm{ET}$ of $5 \mathrm{C}$, cutoff value was $0.308(\mathrm{AUC}=0.94)$, 
right $(\mathrm{C})$ : by using AT/ET of R, cutoff value was 0.331 (AUC = 0.91). Cutoff value for severe AS was larger in $\mathrm{R}$ than in $3 \mathrm{C}$ or $5 \mathrm{C}$.

Figure 7 Inter-observer variability of AT/ET in apical and right parasternal approaches.

Upper raw: Correlogram and Bland-Altman plot of measurements by two observers (A and B) for apical window (3C and 5C) tracings.Lower raw: Correlogram and Bland-Altman plot of measurements by two observers (A and B) for right parasternal window $(\mathrm{R})$ tracings. Correlation coefficient was slightly smaller in $\mathrm{R}(0.826)$ than in $3 \mathrm{C}$ and $5 \mathrm{C}(0.867)$. Coefficient of variation $(\mathrm{CV})$ was slightly greater for the $\mathrm{R}$ approach $(7.92 \%)$ than for the $3 \mathrm{C}$ and $5 \mathrm{C}$ approach $(6.11 \%)$.

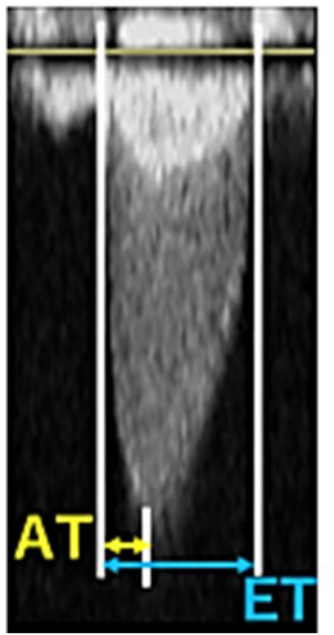

$3 \mathrm{C}$

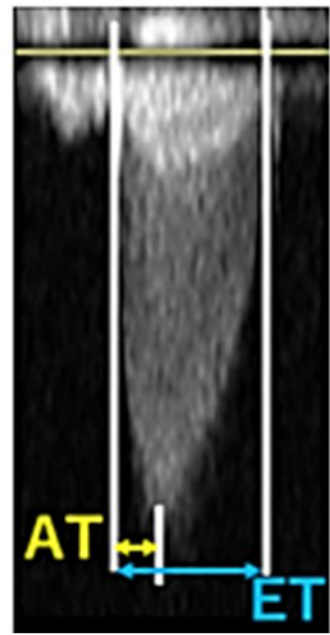

$5 \mathrm{C}$

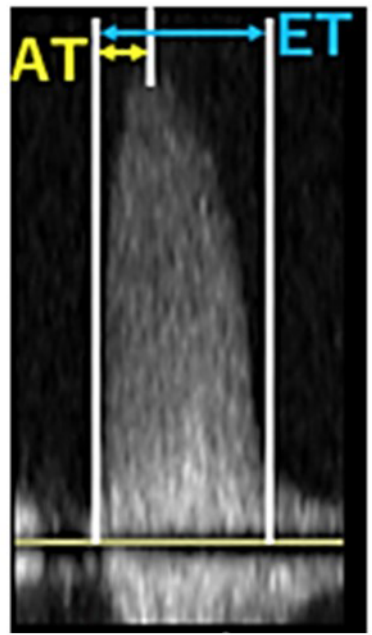

$\mathrm{R}$

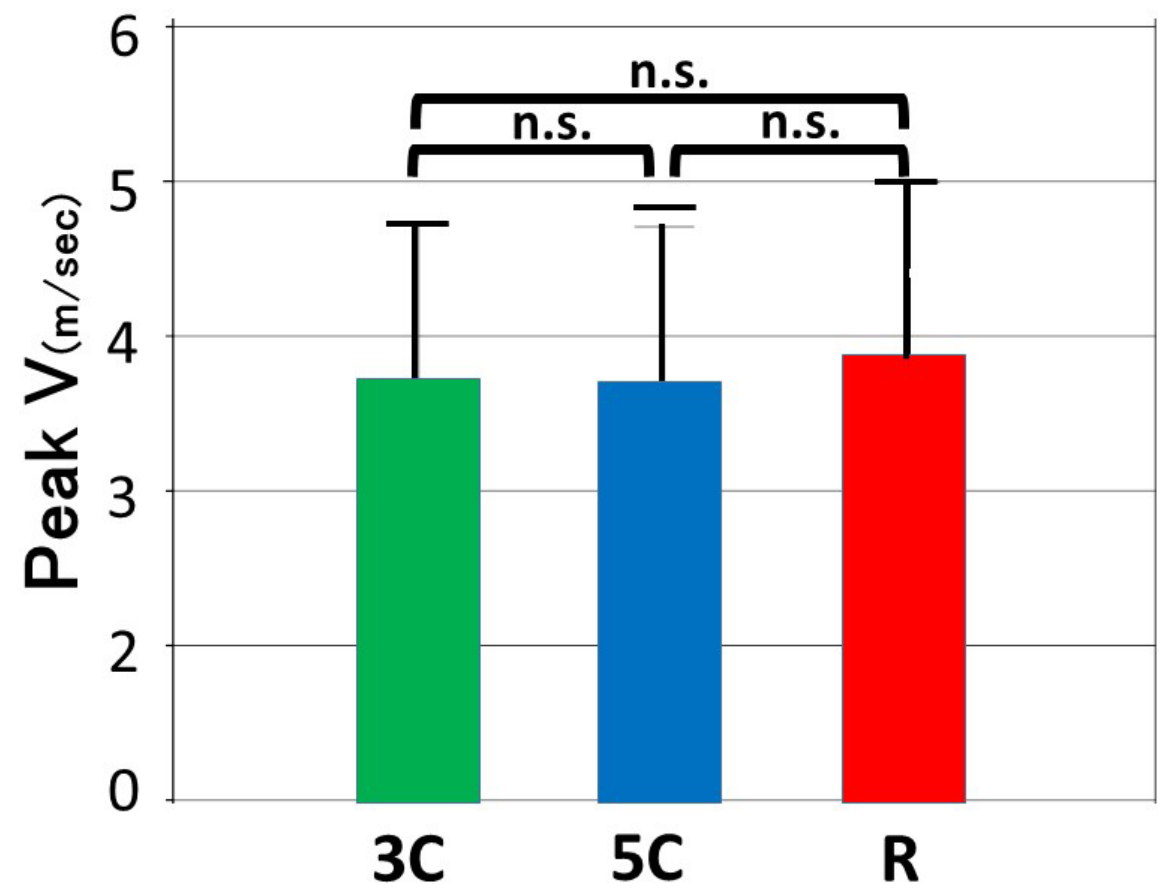



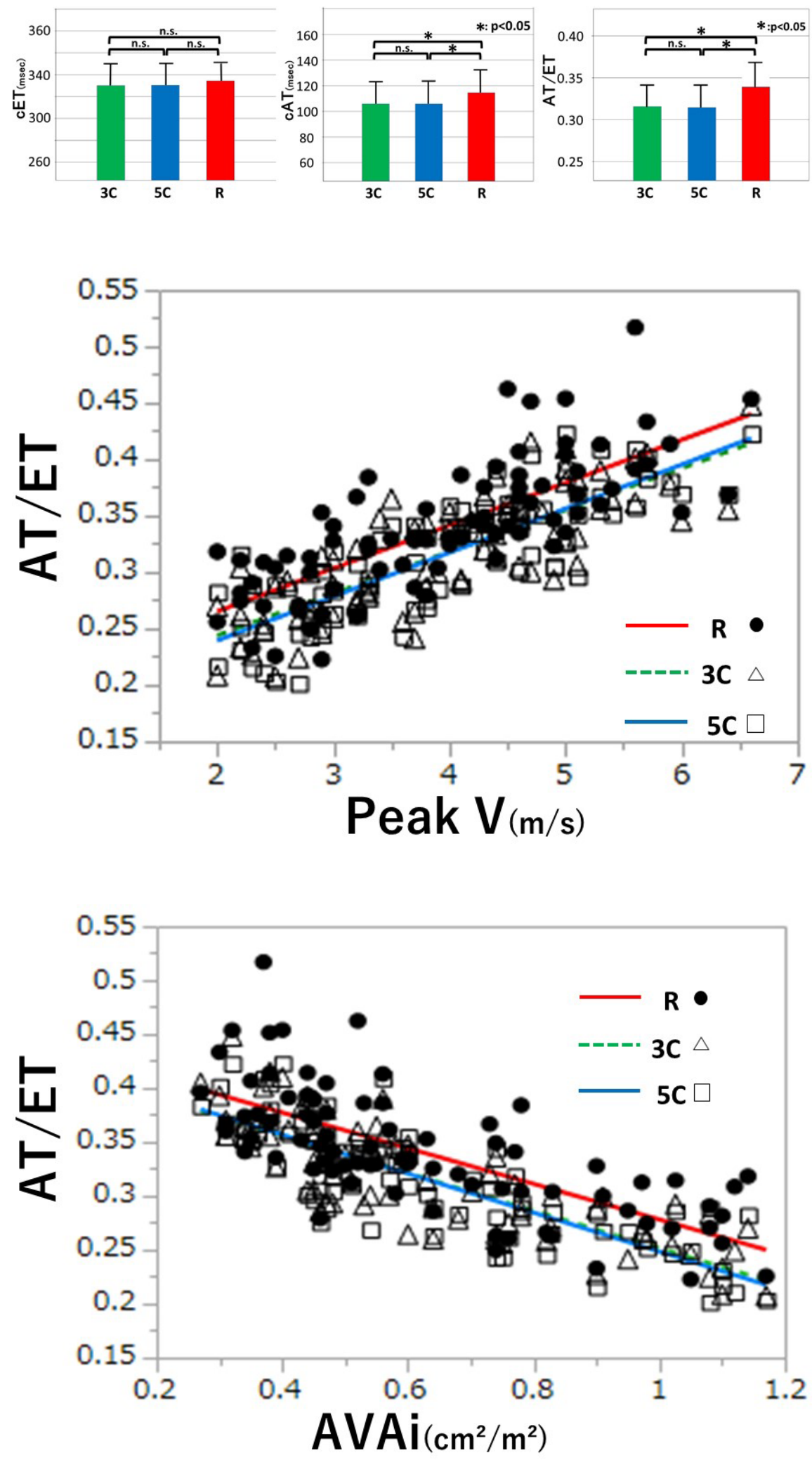

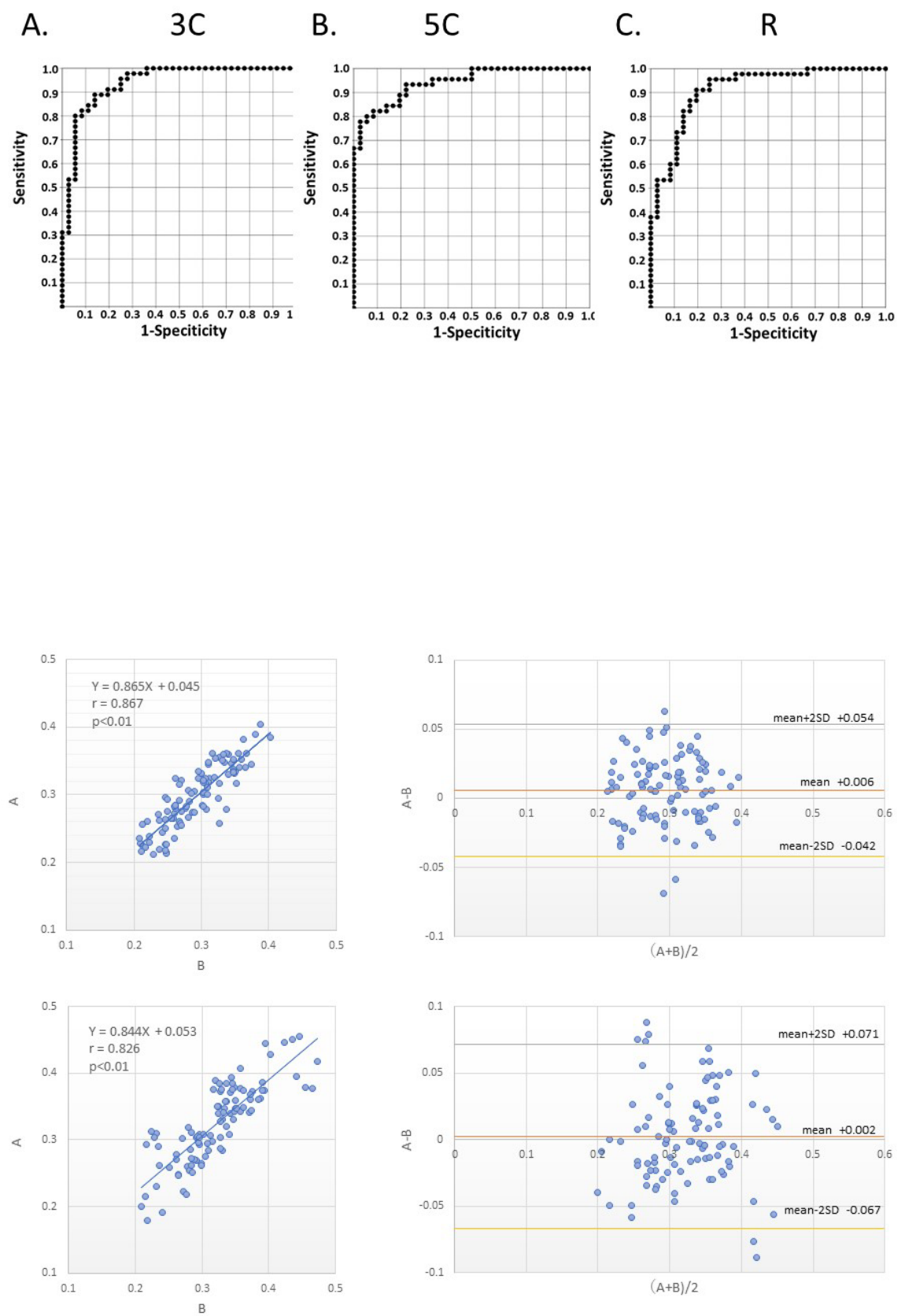